Pacific Journal of Mathematics

DETERMINING A POLYTOPE BY RADON PARTITIONS 


\section{DETERMINING A POLYTOPE BY RADON PARTITIONS}

\section{MARILYN BREEN}

In an extension of the classical Radon theorem, Hare and Kenelly have introduced the concept of a primitive partition, allowing a reduction to minimal subsets which still possess the necessary intersection property.

Here it is proved that primitive partitions in the vertex set $P$ of a polytope reveal the subsets of $P$ which give rise to faces of conv $P$, thus determining the combinatorial type of the polytope. Furthermore, the polytope may be reconstructed from various subcollections of the primitive partitions.

2. Preliminary results. Throughout, $|P|$ denotes the cardinality of $P$. If $P$ is a set of points in $R^{d}, A \cup B$ is a Radon partition for $P$ iff $P=A \cup B, A \cap B=\varnothing$, and conv $A \cap \operatorname{conv} B \neq \varnothing$. Each of $A$ and $B$ is called half a partition for $P$ and each element of $A$ is said to oppose $B$ in the partition. The Radon theorem says that for $P \subseteq R^{d}$ having at least $d+2$ points, there exists a Radon partition for $P$. When $P$ is in general position in $R^{d}$ and $P$ has exactly $d+2$ elements, the partition is unique.

In [2], Hare and Kenelly introduce the concept of a primitive partition: For $P \subseteq R^{d}, A \cup B$ is a Radon partition in $P$ iff $A \cup B$ is a Radon partition for a subset $S$ of $P$. We say that the Radon partition $A \cup B$ extends the Radon partition $A^{\prime} \cup B^{\prime}$ iff $A^{\prime} \leqq A$ and $B^{\prime} \leqq B$. Finally, $A \cup B$ is called a primitive partition in $P$, or simply a primitive, provided it is a Radon partition in $P$ and $A \cup B$ extends the Radon partition $A^{\prime} \cup B^{\prime}$ iff $A^{\prime}=A$ and $B^{\prime}=B$. It is proved that each Radon partition extends a primitive partition having cardinality at most $d+2$.

Theorem 1 follows immediately from the results of Hare and Kenelly.

THEOREM 1. Let $P$ denote a set of $d+2$ points in $R^{d}$ and let $A \cup B$ be a primitive for $P$. Then $|A|+|B|=d+2$ iff $P$ is in general position.

Corollary 1. If $A \cup B$ is a primitive for $P, P \subseteq R^{d}$, then $A \cup B$ is in general position in $R^{k}$ for some $k \leqq d$, and $|A|+|B|=k+2$ for this $k$.

THEOREM 2. If $P \subseteq R^{i}$ and $A \cup B$ is a primitive for $P$, then $\operatorname{dim}(\operatorname{conv} A \cap \operatorname{conv} B)=0$. 
Proof. By the corollary to Theorem 1, $A \cup B$ is in general position in $R^{k}$ for some $k \leqq d$.

Recall that $\operatorname{dim}$ (aff $A \cap$ aff $B$ ) $=\operatorname{dim}$ aff $A+\operatorname{dim}$ aff $B-\operatorname{dim}$ (aff $A+$ aff $B$ ). Letting $j=|A|$ and $l=|B|$, for points in general position, this is equal to $(j-1)+(l-1)-k=j+l-k-2$. Also, for $k+2$ points in general position, the partition is unique, and so $j+l=k+2$, and the above is zero.

3. Reconstructing polytopes. Our goal is to establish the relationship between faces of conv $P$ and primitive partitions for $P$. Throughout, $P$ denotes the vertex set of a convex polytope in $R^{t}$, and $|P|=n$.

THEOREM 3. If $S \subseteq P$ and conv $S$ is a face of conv $P$, then $S$ is not half a Radon partition for $P$.

Proof. Assume conv $S$ is a proper face, for otherwise the result is trivial. Let $H$ be a supporting hyperplane to conv $P$ for which $H \cap \operatorname{conv} P=\operatorname{conv} S$. Assume $P \subseteq \operatorname{cl}\left(H_{+}\right)$, the closure of the open half-space $H_{+}$. Then $P \sim S \subseteq H_{+}$, and conv $(P \sim S) \cap \operatorname{conv} S=\varnothing$.

The following definitions are useful in obtaining a converse to Theorem 3.

Definition. Let $S \subseteq P$. Then we say conv $S$ cuts conv $P$ (or $S$ cuts conv $P$ ) iff one of the following is true: Either (1) dim aff $S=d$ or (2) dim aff $S \leqq d-1$ and any hyperplane containing $S$ cuts conv $P$.

Definition. If $S \subseteq P$ and conv $S$ cuts conv $P$, then a subset $T$ of $S$ is said to be a minimal cutting subset of $S$ for $P$ iff conv $T$ cuts conv $P$ and no subset of $S$ of cardinality less than $|T|$ cuts conv $P$.

Theorem 4. If $|P|=n \geqq d+1$, and $S \varsubsetneqq P$, then the following is true: conv $S$ is a face for conv $P$ iff for $A \subseteq S, A$ is half a primitive for $P$ only in case all the elements opposing $A$ in the primitive are also in $S$.

Proof. If conv $S$ is a face for conv $P$, then by Theorem $3, S$ cannot be half a Radon partition for $P$. Thus if $A \subseteq S$ and $A$ is half a primitive for $P$, some of the elements opposing $A$ must lie in $S$. We must show that all the elements opposing $A$ lie in $S$ :

Suppose not, and let $A \cup B$ be a primitive for $P$ with $A \cong S, B \cap$ 
$S \neq \varnothing$, and $B \cap(P \sim S) \neq \varnothing$. Since $A \cup B$ is a primitive, conv $A \cap$ conv $(B \cap S)$ is empty. Thus any point in conv $A \cap \operatorname{conv} B$ cannot lie in conv $S$. Yet $A \subseteq S$, so conv $A \subseteq$ conv $S$, and we have a contradiction. Our supposition is false, and all members of $B$ lie in $S$.

Conversely, suppose $S \cong P$ has the property that for $A \subseteq S, A$ is half a primitive only in case all the elements opposing $A$ in the primitive come from $S$.

Let $x \in P \sim S \neq \varnothing$.

First we assert that $x \notin$ aff $S$. If $x \in$ aff $S$, then reduce $S$ to a $(k+1)$-subset $T \leqq S$ such that aff $T=\operatorname{aff} S$, where $k=\operatorname{dim}$ aff $S$. Then conv $T$ is necessarily a simplex. Since $T \cup\{x\}$ is a $(k+2)$-subset of $R^{k}=$ aff $(T \cup\{x\})$, there is a Radon partition for $T \cup\{x\}$. Let $A_{0} \cup B_{0}$ be a primitive for $T \cup\{x\}$. Necessarily $x$ appears, since $T$ is a simplex. Assume $x \in B_{0}$. Then $A_{0}$ is a subset of $T$ (and thus a subset of $S$ ) which is half a primitive for $P$. Yet $x$ opposes $A_{0}$ and $x$ is not in $S$, contradicting our hypothesis. Thus we have proved that for $x$ in $P \sim S, x \notin$ aff $S$. Also, this implies that $S=P \cap$ aff $S$ and $\operatorname{dim}$ aff $S \leqq d-1$.

We assert that $S$ lies in a proper face of conv $P$. Assume that $S$ does not lie in a proper face of conv $P$ to reach a contradiction. Let $x \in P \sim S$. If $S$ does not lie in a face of conv $P$, then conv $S$ necessarily cuts conv $P$. Choose $S^{\prime} \subseteq S$ to be a minimal cutting subset of $S$ for $P$. Let $p$ be in conv $S^{\prime}$ and interior to conv $P$. We will show that a subset $A$ of $S^{\prime}$ is half a primitive partition $A \cup B$ for $P$, where $B \nsubseteq S$ :

Consider the ray from $x$ through $p$. Since $p$ is interior to conv $P$, this ray intersects bdry conv $P$ at a point $v$ beyond $p$. Clearly $v \notin$ aff $S$, or else $x \in$ aff $(S \cup\{v\})=$ aff $S$, a contradiction since $x \notin$ aff $S$. Now $v$ lies in a facet $F$ of conv $P$. Choose exactly $d$ vertices $T$ in $F$ such that $v \in$ conv $T$ and $T$ determines a simplex.

Let $Q \equiv T \cup S^{\prime} \cup\{x\}$. Consider the polytope conv $Q$. We will show that $S^{\prime}$ is half a partition for $Q$ :

By minimality of $\left|S^{\prime}\right|$, it follows that aff $S^{\prime} \cap \operatorname{conv} P=\operatorname{conv} S^{\prime}$. For otherwise, conv $S^{\prime}$ is not in a face for the polytope aff $S^{\prime} \cap \operatorname{conv} P$ (since the dimensions are the same), and some proper subset of $S^{\prime}$ must cut aff $S^{\prime} \cap \operatorname{conv} P$. Thus a proper subset of $S^{\prime}$ cuts our original polytope conv $P$, contradicting minimality of $S^{\prime}$. This implies also that aff $S^{\prime} \cap \operatorname{conv} Q=\operatorname{conv} S^{\prime}$.

To show that conv $S^{\prime} \cap \operatorname{conv}\left(Q \sim S^{\prime}\right) \neq \varnothing$, it suffices to show that aff $S^{\prime} \cap \operatorname{conv}\left(Q \sim S^{\prime}\right) \neq \varnothing$. Assume that the intersection is empty to reach a contradiction. If the intersection is empty, then strictly separate aff $S^{\prime}$ from conv $\left(Q \sim S^{\prime}\right)$ by a hyperplane $H$. Since $H \cap$ aff $S^{\prime}=\varnothing, H$ must be parallel to aff $S^{\prime}$. Let $J$ be a hyperplane parallel to $H$ and containing aff $S^{\prime}$. Clearly $J \cap \operatorname{conv}\left(Q \sim S^{\prime}\right)=\varnothing$, so $J$ is a 
supporting hyperplane for conv $Q$ such that $J \cap \operatorname{conv} Q=\operatorname{conv} S^{\prime}$, and $\operatorname{conv} S^{\prime}$ is a face for $\operatorname{conv} Q$. However, this is a contradiction, for the segment $[x, v]$ intersects conv $S^{\prime}$ at $p$. Our assumption is false, conv $S^{\prime} \cap \operatorname{conv}\left(Q \sim S^{\prime}\right)$ is not empty, and $S^{\prime}$ is half a partition for $Q$.

Let $A \cup B$ be a primitive inside $S^{\prime} \cup\left(Q \sim S^{\prime}\right)$. We claim that $x$ necessarily appears in $B$, for otherwise we have $B \cong T$, but conv $T$ is a face for conv $Q$ so by the first part of this theorem, $A \subseteq T$ also. But we chose $T$ to be a simplex, so there is no primitive for $T$; we have a contradiction, and $x$ must appear.

Recall that $x \notin S$. Thus $B \nsubseteq S$ since $x \in B$. At last we have contradicted our hypothesis, for $A \cup B$ is a primitive such that $A \subseteq S$ and $B \nsubseteq S$. Our assumption that $S$ does not lie in a face of conv $P$ is false, and $S$ does indeed lie in a face.

To complete the proof, it remains to show that $\operatorname{conv} S$ is a full face of conv $P$. Select a face $F$ of conv $P$ having minimal dimension for which $S \subseteq F$. Clearly $S$ cannot lie in a proper face of the polytope $F$. Thus, $F \subseteq$ aff $S$, so $P \cap F \subseteq P \cap$ aff $S=S$, and vert $F=S$, finishing the proof.

Corollary 1. For a simplicial polytope conv $P$ and $S \subseteq P$, conv $S$ is a fase for conv $P$ iff no subset of $S$ is half a primitive for $P$.

The proof to Theorem 4 required a construction which we will need again, and for this reason we list it as a corollary:

Corollary 2. Let $S \subseteq P, x \in P \sim$ aff $S \neq \varnothing$. If $S$ does not lie in a face of conv $P$, let $S^{\prime}$ be a minimal cutting subset of $S$ for $P$. Then aff $S^{\prime} \cap \operatorname{conv} P=\operatorname{conv} S^{\prime}$. Moreover, $S^{\prime}$ is half a Radon partition for a subset $Q$ of $P$ where $x \in Q$, and $Q$ may be chosen so that $Q \sim\left[S^{\prime} \cup\{x\}\right]$ is a simplex and lies in a facet of conv $P$. For any primitive $A \cup B$ inside $S^{\prime} \cup\left[Q^{\prime} \sim S^{\prime}\right]$ with $A \leqq S^{\prime}, x \in B$.

Corollary 3. If $P$ is in general position, $S$ half a Radon partition for $P, x \in P \sim S$, and $S^{\prime}$ a minimal cutting subset of $S$ for $P$, then $S^{\prime}$ is half a primitive for $P$, and this primitive may be selected so that $x$ still appears.

Definition. We say that it is possible to reconstruct the polytope conv $P$ iff for each face $F$ of conv $P$ we can determine the unique subset $S$ of $P$ such that conv $S=F$.

The author wishes to thank the referee for the following observation: Let $\mu$ determine the collection of all sets $S \subseteq P$ for which conv $S$ is a face for conv $P$. Since $\mu$ is a complete lattice under inclusion, and each maximal chain in $\mu$ is of length $d+2$, beginning with $\varnothing$ 
and ending with $P$, we can determine the dimension of each face conv $S$ from its position in any maximal chain. The lattice $\mu$ also determines all inclusion relations between faces and hence gives the combinatorial type of conv $P$.

Therefore, when the definition of reconstruct is satisfied, the combinatorial type of the polytope is revealed.

Definition. Let $P_{1}, P_{2}$ be vertex sets for two polytopes conv $P_{1}$, conv $P_{2}$, and let $R_{1}, R_{2}$, denote the set of primitive partitions for $P_{1}, P_{2}$ respectively. We say that $R_{1}$ is isomorphic to $R_{2}$ iff there is a oneto-one map $\psi$ of $P_{1}$ onto $P_{2}$ having the following property: $A \cup B$ is a primitive for $P_{1}$ iff $\psi(A) \cup \psi(B)$ is a primitive for $P_{2}$.

The following corollary is a direct consequence of Theorem 4 .

Corollary 4. Let $P_{1}, P_{2}$ be vertex sets for polytopes, $R_{1}, R_{2}$ their respective primitive partitions. If $R_{1}$ is isomorphic to $R_{2}$, then conv $P_{1}$ is combinatorially equivalent to conv $P_{2}$. Thus it is possible to determine the combinatorial type of a polytope from the Radon partitions of its vertex set.

The following example shows that the converse is false. That is, two polytopes may be combinatorially equivalent although their vertex sets have non-isomorphic Radon partitions.

EXAMPLE 1. Let $\{1,2,3,4\}$ be the vertex set for a square which is base for two distinct bipyramids conv $P_{1}$ and conv $P_{2}$. Let $\{5,6\}$ be the remaining vertices for conv $P_{1}$, and let the segment $[5,6]$ pass through the center of the square. The primitives for $P_{1}$ are

$$
\begin{aligned}
& \{1,3\} \cup\{2,4\}, \\
& \{1,3\} \cup\{5,6\}, \\
& \{2,4\} \cup\{5,6\} .
\end{aligned}
$$

Now let $\{7,8\}$ be the remaining vertices for conv $P_{2}$, where the segment $[7,8]$ intersects the base within $[2,4] \cap$ rel int conv $\{1,2,3\}$. The primitives for $P_{2}$ are

$$
\begin{array}{r}
\{1,3\} \cup\{2,4\} \\
\{1,2,3\} \cup\{7,8\} \\
\{2,4\} \cup\{7,8\} .
\end{array}
$$

The primitives for $P_{1}, P_{2}$ are not isomorphic, yet the map $\psi$ from $P_{1}$ onto $P_{2}$ defined as the identity on $\{1,2,3,4\}, \psi(5)=7, \psi(6)=8$, sets up a one-to-one correspondence between faces and is inclusion preserving. 
Even for points in general position, combinatorial equivalence of conv $P_{1}$, conv $P_{2}$ does not imply that $R_{1}$ is isomorphic to $R_{2}$. However, in case we have exactly $d+2$ points in general position in $R^{d}$, the implication does hold.

Corollary 5. For $i=1,2$, let conv $P_{i}$ be a simplicial polytope having $d+2$ vertices, and let $R_{i}$ be the unique Radon partition for $P_{i}$. Then combinatorial equivalence of conv $P_{1}$, conv $P_{2}$ implies that $R_{1}$ is isomorphic to $R_{2}$.

It is interesting that Corollary 5 may be used to obtain the following familiar result.

CoRollary 6. Consider the collection $\mathscr{P}$ of all sets $P$ in $R^{d}$ consisting of $d+2$ points in general position with no point of $P$ interior to conv $P$. Then there are exactly [d/2] possible Radon partitions for $P$ in $\mathscr{P}$ and each one determines a distinct polytope conv $P$. Therefore, there are exactly [d/2] simplicial polytopes having $d+2$ vertices.

4. Reductions. Of major interest is the problem of obtaining a minimal subcollection of primitive partitions for $P$ which will determine the combinatorial type of conv $P$. The following theorems are concerned with one kind of reduction.

For $x \in P$, let $\mathscr{C}_{x}$ denote the subcollection of primitive partitions for $P$ defined in the following manner: $A \cup B$ belongs to $\mathscr{C}_{x}$ iff either (1) $x$ appears in $A \cup B$ or (2) $|A|+|B| \leqq d+1$.

Theorems 5 and 6 show that conv $P$ may be reconstructed from $\mathscr{C}_{x}$.

THEOREM 5. For $x \in P$ and $S \subseteq P \sim\{x\}$, conv $S$ is not a face for conv $P$ iff there is some member $A \cup B$ of $\mathscr{C}_{x}$ such that $A \subseteq S, B \nsubseteq S$.

Proof. By Theorem 4, if a subset $A$ of $S$ is half a primitive $A \cup B$ for $P$, and $B \nsubseteq S$, conv $S$ cannot be a face for conv $P$.

Conversely, suppose that $x$ is a specified point in $P, S \subseteq P \sim\{x\}$, and conv $S$ is not a face for conv $P$. We consider cases:

Case 1. If $S$ lies in a facet $F$ of conv $P$, then by a fundamental property of polytopes, conv $S$ cannot be a face for $F$. Using Theorem 4 , since conv $S$ is not a face for the polytope $F$, a subset $A$ of $S$ must be half a primitive $A \cup B$ for vert $F$, with $B \nsubseteq S$. Moreover, since $F$ is $(d-1)$-dimensional, $|A|+|B| \leqq d+1$, and Condition (2) is satisfied.

Case 2. If $S$ does not lie in a facet and if $x \in$ aff $S$, then as in the proof of Theorem 4, let $\operatorname{dim}$ aff $S=k \leqq d$ and reduce $S$ to a 
$(k+1)$-subset $T$ of $S$ such that aff $T=$ aff $S$. Conv $T$ is necessarily a simplex. Since $T \cup\{x\}$ is a $(k+2)$-subset of $R^{k}=$ aff $(T \cup\{x\})$, there is a Radon partition for $T \cup\{x\}$. Let $A \cup B$ be a primitive corresponding to this partition. Necessarily $x$ appears since conv $T$ is a simplex. Assume $x \in B$. Then $A \subseteq T \subseteq S$, and Condition (1) is satisfied.

Case 3. If $S$ does not lie in a facet and if $x \notin$ aff $S$, then we may call on the technical corollary following Theorem 4 to obtain a subset $S^{\prime}$ of $S$ and a subset $Q$ of $P$ having the property that $S^{\prime} \cup$ $\left(Q \sim S^{\prime}\right)$ is a Radon partition for $Q$. Moreover, if $A \cup B$ is a primitive inside $S^{\prime} \cup\left(Q \sim S^{\prime}\right)$, then $x$ appears in $B$. Thus $A \subseteq S, B \nsubseteq S$, and $x$ opposes a subset of $S$ in this primitive. We have satisfied Condition (1) and completed the proof of the theorem.

For $x$ in $P$, Theorem 5 allows us to recognize all faces of conv $P$ not containing $x$ by listing the primitives in which $x$ appears plus the primitives having $\leqq d+1$ points. Our next problem, of course, is recognizing the faces containing $x$, and we would like to be able to do this from the same collection of primitives. Happily, the next theorem shows that this is possible.

THEOREM 6. For $T \varsubsetneqq P$ and $x$ in $T$, conv $T$ is not a face for conv $P$ iff there is some member $A \cup B$ of $\mathscr{C}_{x}$ such that $A \subseteq T, B \nsubseteq T$.

Proof. Certainly if there is a primitive $A \cup B$ with $A \subseteq T$ and $B \nsubseteq T$, then by Theorem 4, conv $T$ cannot be a face for conv $P$.

Conversely, assume that conv $T$ is not a face for conv $P$ and $x \in T$. Again, we must consider cases:

Case 1. Now if $T$ lies in a facet $F$ of conv $P$, repeating the argument in Case 1 of Theorem 5 shows that Condition (2) is satisfied.

In the remaining cases, assume that $T$ does not lie in a facet for conv $P$. Let $S \equiv T \sim\{x\}$ :

Case 2. If $S$ is contained in a facet $F$ but conv $S$ is not a face for conv $P$, then by repeating the argument in Case 1 of Theorem 5, Condition (2) holds.

Case 3. Suppose $S$ is contained in a facet and conv $S$ is a face for conv $P$. Recall $T \equiv S \cup\{x\}$ is not a face, for we are assuming that $T$ does not lie in a facet. By Theorem 4, there is a primitive $A \cup B$ for $P$ with $A \subseteq S \cup\{x\} \equiv T$ and $B \nsubseteq S \cup\{x\}$. Moreover, since conv $S$ is a face for conv $P$, a subset $C$ of $S$ is half a primitive $C \cup D$ for $P$ iff $D \leqq S$. This implies that $x$ must appear in $A$, for otherwise 
we would have $A \subseteq S$ and $B \nsubseteq S$, a contradition. Thus $A \subseteq T, B \nsubseteq T$, and $x$ appears, satisfying Condition (1).

Case 4. If conv $S$ is not in a facet for $\operatorname{conv} P$ and $x$ is in aff $S$, then unfortunately it is necessary to consider subcases:

(4a) If $\operatorname{dim}$ aff $S=d$, then since $T \neq P$, there is some $y \in P \sim T$ and necessarily $y$ is in aff $S$. Let $T^{\prime}$ be the vertex set for a $d$-dimensional simplex, $x \in T^{\prime} \subseteq T \equiv S \cup\{x\}$. Then $T^{\prime} \cup\{y\}$ is a set having $d+2$ points in $R^{t}$, so there is a primitive $A \cup B$ for $T^{\prime} \cup\{y\}$. Certainly $y$ appears (since $T^{\prime}$ is a simplex). Assume $y \in B$. Then $A \leqq T^{\prime} \leqq T$, and $B \nsubseteq T$. Now if $|A|+|B|=d+2$, then $x$ appears and Condition (1) holds. If $|A|+|B| \leqq d+1$, then Condition (2) holds.

(4b) Similarly, if $\operatorname{dim}$ aff $S=k<d$ and if there is some $y$ in $(P \cap$ aff $S) \sim T$, let $T^{\prime}$ be the vertex set for a $k$-dimensional simplex, $x \in T^{\prime} \subseteq T$, and repeat the above proof.

(4c) If $\operatorname{dim}$ aff $S=k<d$ and if $(P \cap$ aff $S) \sim T=\varnothing$, then select a point $y \in P \sim$ aff $S$. (This is possible since $T \neq P$.) Again, let $T^{\prime}$ be the vertex set for a $k$-dimensional simplex, $x$ in $T^{\prime} \subseteq T$.

Now we want to use our old friend, the corollary following Theorem 4, but first we must make a few adjustments.

Let $\operatorname{conv} R$ be a new polytope, where $R \equiv P \sim$ (aff $T \sim T^{\prime}$ ). We have thrown away the vertices in aff $T$ except for those in $T^{\prime}$. Notice that $x$ remains. Also $y$ remains since $y \notin$ aff $S=$ aff $T$.

We assert that $T^{\prime}$ does not lie in a face of $\operatorname{conv} R$ : If $T^{\prime}$ is in a face, then let the hyperplane $H$ support conv $R$ with $T^{\prime} \leqq H$. Then aff $T^{\prime} \leqq H$. But aff $T^{\prime}=$ aff $T$, so aff $T \subseteq H$, and $H$ supports conv $P \equiv \operatorname{conv}(R \cup T)$ with $T \subseteq H$. But $T$ does not lie in a face of conv $P$ by hypothesis. We have a contradiction, and $T^{\prime}$ does not lie in a face of conv $R$.

We are ready for the corollary to Theorem 4. $T^{\prime}$ does not lie in a face of conv $R$, and $y$ is in $R \sim$ aff $T^{\prime}$. Thus there is a subset $T^{\prime \prime}$ of $T^{\prime}$ which appears as half a Radon partition for a subset $Q$ of $R$, where $y \in Q$. Moreover, $Q$ may be chosen so that $Q \sim\left(T^{\prime \prime} \cup\{y\}\right)$ is a simplex and lies in a facet of $\operatorname{conv} R$. For any primitive $A \cup B$ inside $T^{\prime \prime} \cup\left(Q \sim T^{\prime \prime}\right)$ with $A \subseteq T^{\prime \prime}, y \in B$.

Now if $x$ is in $T^{\prime \prime}$, and if $x \in A$, then we have $A \subseteq T, B \nsubseteq T$ (since $y \in B$ ), and $x$ appears in the primitive, satisfying Condition (1). If $x$ is in $T^{\prime \prime}$ but $x$ is not in $A$, then by our minimality condition of $T^{\prime \prime}$, no proper subset of $T^{\prime \prime}$ may cut conv $R$, so conv $A$ cannot cut conv $R$, and likewise, conv $A$ cannot cut conv $Q$. Then conv $A$ must lie in some face of conv $Q$, and certainly conv $A \cap \operatorname{conv} B$ must lie in the boundary of conv $Q$. By Theorem 1, Corollary 1, necessarily $|A|+$ $|B| \leqq d+1$, satisfying Condition (2).

We still need to examine what happens in case $x$ does not appear 
in $T^{\prime \prime}$. Again by the corollary to Theorem 4, aff $T^{\prime \prime} \cap \operatorname{conv} R=\operatorname{conv} T^{\prime \prime}$. Now conv $T^{\prime}$ is a simplex, $T^{\prime \prime} \leqq T^{\prime}$, and $x \in T^{\prime}$. If $x$ is not in $T^{\prime \prime}$, then $x \notin \operatorname{conv} T^{\prime \prime}$, and so $x \notin$ aff $T^{\prime \prime}$. By the very choice of $T^{\prime \prime}$, conv $T^{\prime \prime}$ cuts conv $R$, and so conv $T^{\prime \prime}$ does not lie in a face of conv $R$. Also $\imath \in R \sim \operatorname{aff} T^{\prime \prime}$, so there is a subset $T^{(3)}$ of $T^{\prime \prime}$ which is half a partition for a subset of $R$ (by the corollary). Let $C \cup D$ be a corresponding primitive. Then $C \leqq T^{(3)}$ and $x \in D$. Not all of $D$ can lie in $T^{\prime}$, for if it did, we would have a primitive $C \cup D$ in the vertex set of the simplex $T^{\prime}$, and this is ridiculous. Thus, $D \nsubseteq T^{\prime}$, but $D \subseteq R$, and the only points of $T$ in $R$ are those in $T^{\prime}$. Thus, $D \nsubseteq T$. To review, $C \subseteq T, D \nsubseteq T$, and $x$ appears in $D$, satisfying Condition (1), and completing Case $4 \mathrm{c}$.

Case 5. If $S$ is not in a face and $x$ is not in aff $S$, then as in Case $4 \mathrm{c}$, reduce conv $P$ to a new polytope conv $R$, where $R \equiv P \sim$ (aff $S \sim S^{\prime}$ ), and where $S^{\prime}$ is the vertex set for a $k$-dimensional simplex with $k=\operatorname{dim}$ aff $S$. By our earlier argument, $S^{\prime}$ does not lie in a face of conv $R$. Also, $x \in R$ and $x \notin$ aff $S^{\prime}$. Then by the corollary to Theorem 4, a subset $S^{\prime \prime}$ of $S^{\prime}$ appears as half a partition for a subset $Q$ of $R$. Let $A \cup B$ be a corresponding primitive. Then by the corollary, $A \subseteq S^{\prime \prime}$ and $x \in B$. Moreover, $B \nsubseteq T \equiv S \cup\{x\}$, for if $B \subseteq T$, we would have $A \subseteq S^{\prime}, B \subseteq T \cap Q \equiv S^{\prime} \cup\{x\}$. But $S^{\prime}$ determines a simplex and $x \notin$ aff $S^{\prime}$, so $S^{\prime} \cup\{x\}$ determines a simplex and has no primitives. Thus $A \subseteq T, B \nsubseteq T$, and $x$ appears in $B$, satisfying Condition (1) and finishing Case 5 .

This completes the proof of Theorem 6 .

At last we have obtained a reduction in the number of partitions necessary to reconstruct an arbitrary polytope. Combining Theorems 5 and 6 , we have the following corollaries:

Corollary 1. The combinatorial type of conv $P$ is determined by $\mathscr{C}_{x}$ for any $x \in P$.

Corollary 2. For $P$ in general position and $x \in P$, the combinatorial type of conv $P$ is determined by the primitive partitions for $P$ which contain $x$.

5. Locating points. Another approach to the problem of obtaining a minimal collection of primitive partitions which determine conv $P$ leads to the method of reconstructing a polytope by locating vertices, one at a time.

Definition. Let $P \cup\{x\}$ be the vertex set for a polytope in $R^{l}$ and assume that we have reconstructed conv $P$. We say that we 
locate $x$ relative to conv $P$ iff we are able to reconstruct conv $(P \cup\{x\})$.

Definition. Let $P$ be the vertex set for a polytope in $R^{d}$ and let $x$ be a point not in $P$. For $F$ a facet of $\operatorname{conv} P$, we say $x$ is beyond $F$ iff $x$ is in the open halfspace of $H_{F}$ not containing $P$ (where $H_{F}$ is the hyperplane determined by $F$ ). For $E$ a face of conv $P$, we say $x$ is beyond $E$ iff $x$ is beyond $F$ for every facet $F$ containing $E$.

To reconstruct conv $P$ by locating vertices, one at a time, first select a $(d+1)$-subset $S$ of $P$ for which there is no primitive. (Clearly $S$ determines a simplex.) The following theorem describes the procedure for locating additional points.

Theorem 7. Let $P \cup\{x\}$ be the vertex set for a polytope, and assume that we have reconstructed conv $P$. Then to reconstruct conv $(P \cup\{x\})$, it is sufficient to consider the primitives $A \cup B$ for $P \cup\{x\}$ such that $A$ lies in a face of conv $P, x \in B$, and $x$ opposes no proper subset of $A$ in a primitive.

Proof. Using Theorem 5.2.1 of Grünbaum [1], we see that to establish the faces for conv $(P \cup\{x\})$, it suffices to examine the faces for conv $P$.

For $S \subseteq P$ and conv $S$ a face for conv $P, S$ determines a face for conv $(P \cup\{x\})$ iff no subset $A$ of $S$ appears as half a primitive $A \cup B$ with $x$ in $B$. Also, $S \cup\{x\}$ determines a face for conv $(P \cup\{x\})$ iff for every primitive $A \cup B$ with $A \subseteq S$ and $x$ in $B$, then $B \subseteq S \cup\{x\}$.

However, if there is one primitive $A_{0} \cup B_{0}$ with $A_{0} \subseteq S, x \in B_{0}$, and $B_{0} \subseteq S \cup\{x\}$, then by general position of the points involved, $x \in$ aff $S$, $x$ lies in every face containing $S$, and $S \cup\{x\}$ determines a face for $\operatorname{conv}(P \cup\{x\})$. Therefore, if one primitive with $A_{0} \leqq S$ and $x$ in $B_{0}$ satisfies $B_{0} \subseteq S \cup\{x\}$, then every primitive with $A \subseteq S$ and $x$ in $B$ satisfies $B \cong S \cup\{x\}$, and it is easy to determine all faces of conv ( $P \cup$ $\{x\})$ from those listed.

As the following example illustrates, the construction in Theorem 7 allows us to locate $x$ relative to conv $P$ but does not allow us to locate $x$ relative to $\operatorname{conv} Q$, where $Q \subsetneq P$.

ExAMPLE 2. Let $\{1,2\} \cup\{3,4,5\}$ be the primitive partition for the set $P=\{1,2,3,4,5\}$ in $R^{3}$, and let 6 lie beyond the face conv $\{1,4,5\}$. This does not determine the location of 6 relative conv $Q, Q=\{1,2,3,4\}$, for 6 may or may not lie beyond the edge $[1,2]$ of $\operatorname{conv} Q$.

REMARK. It is easy to find examples for which the subcollection of primitive partitions described in Theorem 7 is minimal. Moreover, at each stage of the construction at least one primitive is required 
to locate an additional vertex. Thus at least $n-(d+1)$ primitive partitions are needed to reconstruct conv $P$. This lower bound is always attained for simplicial polytopes having $d+2$ vertices.

\section{REFERENCES}

1. Branko Grunbaum, Convex Polytopes, New York, 1967.

2. William R. Hare and John W. Kenelly, Characterizations of Radon partitions, Pacific J. Math. 36 (1971), 159-164.

3. J. Radon, Mengen konvexer Korper, die einem gemeinsamen Punkt enthalten, Math. Ann., 83 (1921), 113-115.

Received July 20, 1971 and in revised form December 16, 1971.

Clemson UNIVERSITY

AND

UNIVERSITY OF OKLAHOMA 



\section{PACIFIC JOURNAL OF MATHEMATICS}

\section{EDITORS}

\section{H. SAMElson}

Stanford University

Stanford, California 94305

C. R. Новву

University of Washington

Seattle, Washington 98105
J. DugunduI

Department of Mathematics University of Southern California

Los Angeles, California 90007

RICHARD ARENS

University of California

Los Angeles, California 90024

\section{ASSOCIATE EDITORS}
E. F. BECKENBACH
B. H. NeUmanN
F. WOLF
K. YosHIDA

\section{SUPPORTING INSTITUTIONS}

UNIVERSITY OF BRITISH COLUMBIA

UNIVERSITY OF SOUTHERN CALIFORNIA

CALIFORNIA INSTITUTE OF TECHNOLOGY

STANFORD UNIVERSITY

UNIVERSITY OF CALIFORNIA

UNIVERSITY OF TOKYO

MONTANA STATE UNIVERSITY

UNIVERSITY OF UTAH

UNIVERSITY OF NEVADA

WASHINGTON STATE UNIVERSITY

NEW MEXICO STATE UNIVERSITY

OREGON STATE UNIVERSITY

UNIVERSITY OF OREGON

OSAKA UNIVERSITY

UNIVERSITY OF WASHINGTON

* *

AMERICAN MATHEMATICAL SOCIETY

NAVAL WEAPONS CENTER 


\section{Pacific Journal of Mathematics}

\section{Vol. 43, No. $1 \quad$ March, 1972}

Alexander (Smbat) Abian, The use of mitotic ordinals in cardinal

arithmetic ....................................... 1

Helen Elizabeth. Adams, Filtrations and valuations on rings ......... 7

Benno Artmann, Geometric aspects of primary lattices .............. 15

Marilyn Breen, Determining a polytope by Radon partitions ........... 27

David S. Browder, Derived algebras in $L_{1}$ of a compact group .......... 39

Aiden A. Bruen, Unimbeddable nets of small deficiency .............. 51

Michael Howard Clapp and Raymond Frank Dickman, Unicoherent

compactifications ............................... 55

Heron S. Collins and Robert A. Fontenot, Approximate identities and the strict topology ................................... 63

R. J. Gazik, Convergence in spaces of subsets................. 81

Joan Geramita, Automorphisms on cylindrical semigroups ........... 93

Kenneth R. Goodearl, Distributing tensor product over direct product ..... 107

Julien O. Hennefeld, The non-conjugacy of certain algebras of

operators ................................... 111

C. Ward Henson, The nonstandard hulls of a uniform space ........... 115

M. Jeanette Huebener, Complementation in the lattice of regular

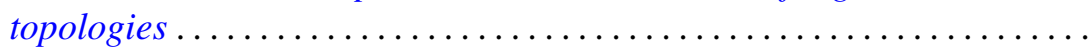

Dennis Lee Johnson, The diophantine problem $Y^{2}-X^{3}=A$ in a

polynomial ring .................................... 151

Albert Joseph Karam, Strong Lie ideals . . . . . . . . . . . . . . . . . . . . 157

Soon-Kyu Kim, On low dimensional minimal sets ............... 171

Thomas Latimer Kriete, III and Marvin Rosenblum, A Phragmén-Lindelöf

theorem with applications to $M(u, v)$ functions ..... . .

William A. Lampe, Notes on related structures of a universal algebra . . . . 189

Theodore Windle Palmer, The reducing ideal is a radical .

207

Kulumani M. Rangaswamy and N. Vanaja, Quasi projectives in abelian and module categories ................................ 221

Ghulam M. Shah, On the univalence of some analytic functions ......... 239

Joseph Earl Valentine and Stanley G. Wayment, Criteria for Banach

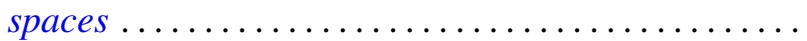

Jerry Eugene Vaughan, Linearly stratifiable spaces ............... 253

Zbigniew Zielezny, On spaces of distributions strongly regular with respect to partial differential operators ..................... 